\title{
La tecnología, una estrategia de innovación educativa para los niños de preescolar aprobada por los maestros y padres de familia
}

\author{
Mónica María Córdoba Castrillón ' \\ Javier Ospina Moreno ${ }^{2}$
}

Recibido: 04-09-2019

Aceptado: 08-05-2020

\section{Resumen}

El preescolar va desde los 3 hasta los 5 años, periodo en que el niño se interesa por descubrir, es curioso, necesita conocer y aprender. A partir de esta etapa el ser humano puede aprender el uso adecuado de las mediaciones tecnológicas, vistas como herramientas de apoyo, como una estrategia innovadora educativa que permitirá que los hombres del futuro puedan aprovechar las bondades del mundo digital.

El objetivo de la investigación es evidenciar cómo el uso de la tecnología puede resultar una estrategia de innovación educativa para el aprendizaje de los niños. La orientación del diseño metodológico de la investigación corresponde a un estudio descriptivo-cuantitativo, no experimental, con toma de datos longitudinal; se seleccionó una institución educativa pública del municipio de Bello (Antioquia) con nivel preescolar; para la muestra

\footnotetext{
1. Administradora de empresas, especialista en Gerencia Educacional, magíster en Administración de Organizaciones, Corporación Universitaria Remington, Medellín, Colombia.

Correo electrónico: monica.cordoba@uniremington.edu.co

ORCID: https://orcid.org/0000-0002-8965-1146

Google Scholar: https://scholar.google.es/citations?user=VoW_OkMAAAAJ\&hl=es

2. Ingeniero de Sistemas, especialista en Gerencia de Sistemas, magíster en Gestión de la Tecnología Educativa, Corporación Universitaria Remington, Medellín, Colombia. Correo electrónico: javier.ospina@uniremington.edu.co

ORCID: https://orcid.org/0000-0002-4168-3915

CvLac: https://scienti.minciencias.gov.co/cvlac/visualizador/generarCurriculoCv. do?cod_rh=0001298046
} 
se seleccionaron acudientes, con nivel de confianza del $95 \%$ que arrojó como resultado 67; para los expertos infantiles, una muestra definida por conveniencia con un total de 50 especialistas, y los maestros directores de los grupos.

Los resultados muestran aprobación por maestros, padres y especialistas infantiles con respecto al uso de la tecnología con impacto positivo y con una debida supervisión; esta realmente es una estrategia efectiva, innovadora en el proceso de aprendizaje en los niños de preescolar.

Palabras clave: preescolar, juego, familia, mediaciones tecnológicas, innovación educativa.

\section{Technology, an educational innovation strategy for preschool children approved by teachers and parents}

\section{Abstract}

Preschool goes from 3 to 5 years old, a period in which the child is interested in discovering, is curious, needs to know and learn. From this stage, human beings can learn the proper use of technological mediations, seen as support tools, as an innovative educational strategy that will allow men of the future to take advantage of the benefits of the digital world.

The objective of the research is to show how the use of technology can be an educational innovation strategy for children's learning. The orientation of the methodological design of the research corresponds to a descriptive-quantitative, non-experimental study, with longitudinal data collection; A public educational institution in the municipality of Bello (Antioquia) with a preschool level 
was selected; For the sample, caregivers were selected, with a confidence level of $95 \%$ that resulted in 67 ; for the child experts, a sample defined by convenience with a total of 50 specialists, and the teacher-directors of the groups.

The results show approval by teachers, parents and child specialists regarding the use of technology with positive impact and with proper supervision; This really is an effective, innovative strategy in the learning process in preschool children.

Keywords: preschool, game, family, technological mediations, educational innovation.

\section{Introducción}

Es una realidad universal que los niños de hoy son diferentes a los de generaciones anteriores, incluso sus juegos son diferentes; observar hoy a un pequeño manipulando un dispositivo digital, como celulares, tabletas o juguetes digitales, es algo cotidiano y común, pues nacieron en la era donde lo digital es lo usual, de ahí que los que conforman las nuevas generaciones sean clasificados como nativos digitales. Pero al igual que los niños de generaciones anteriores necesitan de excelentes procesos formativos, y de que los padres, los maestros y la sociedad en general estén atentos a vigilar lo que es bueno y malo para ellos.

El preescolar es aquella etapa del proceso educativo que antecede a la formación primaria, y va desde los 3 a los 5 años; es el periodo en el cual el niño se interesa completamente por descubrir, todo le causa curiosidad y necesita conocer, aprender. Durante esta etapa experimentan un desarrollo extraordinario; y es desde esta edad cuando padres, maestros y toda la sociedad pueden empezar a formar hombres de bien, es decir, seres humanos capaces de vivir en comunidad, teniendo en cuenta todas las dimensiones 
del desarrollo humano. A partir de esta etapa el ser humano puede aprender a hacer uso adecuado de las mediaciones tecnológicas vistas como herramientas de apoyo y estrategia innovadora educativa que permitirá que los hombres del futuro puedan aprovechar las bondades del mundo digital.

Para apoyar la investigación se utilizaron conceptos acerca del preescolar, el juego en la etapa del preescolar, el papel de la familia en el preescolar y el impacto de la presencia de las mediaciones tecnológicas en la etapa preescolar y las tecnologías como innovación educativa en el preescolar desde el punto de vista de diferentes autores. En general, para muchos autores el preescolar es aquella etapa del ser humano que va desde los 3 a los 5 años, es una etapa en la que el ser humano experimenta y conoce realmente lo que está a su alrededor, es una etapa en la cual las dimensiones del ser humano se desarrollan para alcanzar un desenvolvimiento autónomo y de libertad. En esta etapa es primordial el acompañamiento por parte de los padres y maestros y de la sociedad en general que tenga que ver con el proceso formativo del niño.

Es una necesidad entonces entender que nuestras nuevas generaciones actuales y futuras se enfrentan a un mundo donde la tecnología avanza de forma vertiginosa, pero es nuestro deber prepararlos para aprovechar de forma efectiva las bondades que trae consigo la era digital. Padres, maestros y expertos infantiles avalan completamente el uso de mediaciones tecnológicas como estrategias de innovación educativa en la etapa preescolar, teniendo en cuenta que debe hacerse con precaución y debida supervisión; el uso de software como videojuegos resulta inofensivo en el proceso de aprendizaje de los niños del nivel de preescolar, no es malo, por el contrario, es motivador e innovador.

Con el desarrollo de la investigación se pretende demostrar y evidenciar que a través de la lúdica apoyada de las herramientas tecnológicas como plataformas, páginas web, videos, videojuegos el aprendizaje de los niños de preescolar puede seguir siendo significativo, y además puede convertirse en un proceso variado, motivacional, en el que la familia pueda tener una participación activa; 
y en el cual se fomente el crecimiento personal y comunitario, y se recupere la promoción de valores y principios sociales que hagan de estos niños hombres capaces de luchar por una sociedad sana y tranquila.

\section{Marco térico}

El desarrollo tecnológico es un proceso que avanza de forma acelerada y que aporta significativamente a todas las áreas del conocimiento; sin importar la edad hoy el uso de dispositivos de comunicación digital es tan común tanto en adultos, como en jóvenes e incluso niños. El objetivo de esta investigación es mostrar cómo desde el campo educativo, iniciando desde el nivel preescolar con la utilización de un simple juego o videojuego enmarcado en una plataforma educativa, se puede aprovechar el potencial que tienen las herramientas tecnológicas en los procesos de enseñanzaaprendizaje a temprana edad, y de esta forma generar una cultura de uso efectivo de medios y mediaciones tecnológicas en las generaciones digitales.

Para el éxito de la investigación fue necesario abordar teorías que permitieran mostrar cómo desde la metodología del juego los infantes digitales pueden aprovechar de forma positiva el uso de plataformas, videos, páginas web o videojuegos bajo la supervisión de padres y maestros para sus procesos de aprendizaje significativo. Para este proceso fue necesario abordar nociones acerca de los siguientes temas.

\section{La etapa preescolar}

El preescolar es aquella etapa que antecede al proceso de formación denominado-primaria, es el primer nivel del sistema educativo colombiano y contempla los grados de prejardín, jardin y transión. Es una etapa de adaptación del niño al ambiente escolar y de inde- 
pendencia ya que son capaces de realizar solos algunas actividades de su desarrollo personal. Laureano (2015) plantea:

La etapa preescolar es un periodo que abarca de los 3 a 5 años y constituye el final de la primera infancia. Es una edad de descubrimiento, inventos, curiosidades y de desarrollo de tipos de conducta sociocultural. De alguna forma constituye un momento más fácil y cómodo para los padres, en especial cuando el niño consigue dominar algunas tareas del desarrollo, la independencia y el cuidado de sí mismo. Los logros biológicos, psicosociales, cognitivos, morales, espirituales y sociales de los preescolares los preparan para el cambio más importante de su estilo de vida: la entrada a la escuela (p.24).

El ser humano, para su sano desarrollo, requiere desde temprana edad una adecuada estimulación, que le permita un crecimiento saludable no solo en el aspecto físico sino también a en el cognitivo, es por esto la importancia que tiene el proceso que se vive con los niños en la etapa del preescolar. Carrero y Martínez (2014) plantean que "entre los 3 a 6 años, los niños experimentan un desarrollo extraordinario de sus habilidades y motivaciones, para pensar acerca de lo que hacen, predecir el resultado de sus acciones, el lenguaje y recordar sus experiencias mostrando que las vivencias que adquieren en el preescolar son significativas para su crecimiento integral" (p.6). Durante su vivencia en esta etapa formativa el niño desarrolla habilidades como: Expresión coherente de sus ideas, uso de oraciones completas, habilidades para describir láminas, objetos y fenómenos, mejor pronunciación de palabras, expresión de sus sentimientos, en todos los campos culturales sociales. El niño aprende la importancia de compatir, de respetar a sus compañeros, maestros y personas que lo rodean, empieza a entender las implicaciones de algunas acciones y puede iniciar con el proceso de toma de decisiones acordes a su edad.

Un sistema educativo exitoso es aquel que se centra y se dedica con calidad a procesos formativos desde los primeros años formativos, ya que permite que los niños pasen por esa etapa de 
adaptación y preparación, antes de iniciar la primaria y poder experimentar una etapa de exploración y acoplamiento ya que desprenderse del ambiente materno puede ser traumático para ellos. Según Cardemil y Román (2014):

Los apoyos y espacios formativos dados a los niños y niñas durante los primeros años, permitirán (o no) que ellos desarrollen sus talentos, aptitudes y capacidades futuras. Debido a ello no resulta ni sorpresivo ni casual, que la asistencia a la Educación Infantil sea una variable de alta significación en la explicación del éxito escolar en los primeros años de Educación Básica. Los niños/as que han accedido a una educación en la etapa preescolar, llegan mejor preparados para enfrentar los procesos y desafíos escolares que supone la etapa obligatoria y el sistema formal, que aquellos que no han pasado por dicha experiencia (p.9).

El filósofo Pitágoras afirmaba: "Educa a los niños y no será necesario castigar a los hombres"; esta máxima puede relacionarse directamente con el tema en cuestión: si desde los primeros años de vida del individuo se dedica el tiempo suficiente para iniciar los procesos formativos con calidad se puede asegurar tener en el futuro hombres de bien, adaptados correctamente a cada etapa siguiente. Para Cardemil y Román (2014):

Los propósitos de la educación temprana, se hacen cargo de la necesidad y del sentido que adquieren estos procesos formativos a esas edades: asegurar el pleno e igualitario acceso al conocimiento de los niños y las niñas. Ampliando de este modo, sus capacidades y habilidades cognitivas al tiempo que ofreciendo espacios y experiencias de aprendizajes que permitan el desarrollo de las dimensiones social, emocional, cívica, ética y moral de ellos. (p.9).

En estos primeros años formativos el niño aprende cada una de las partes de su cuerpo y la importancia del autocuidado, de igual 
forma aprende a entender sus sentimientos y como comunicarlos a otros. En el nivel preescolar cada uno de sus grados tiene un enfóque específico para el desarrollo de las dimensiones del ser humano preparando al niño para el ingreso efectivo a los procesos siguientes a la escolaridad, según Cardemil y Román (2014):

La Educación Preescolar abarca además del desarrollo de estas habilidades, otras en áreas como el conocimiento de sí mismo y de su entorno natural y cultural, la corporalidad en los aspectos de sensorialidad y coordinación, expresividad de sentimientos y comunicación, estimulación en aptitudes creativas originales, así como la socialización en relación con la interiorización de normas y valores entre otros. (p.10.)

En esta estapa del preescolar el papel de la familia y de la escuela es fundamental en el correcto desarrollo de los niños, ya que si cumplen al pie de la letra con la planeación de acciones que conlleven a una verdadera etapa de adaptación y preparación y además puedan rodearlo de un clima saludable y un ambiente familiar adecuado, el niño responderá de forma efectiva el proceso de aprendizaje. Para Carrero y Martínez (2014):

El niño que interactúa saludablemente adquiere valores de sociabilidad y se ve favorecido para el desarrollo cognitivo en el proceso de aprendizaje. Por lo tanto, en la educación del menor ejerce igual influencia tanto la escuela como la familia puesto que en ambos contextos se propicia la educación (p.6).

Se hace referencia entonces a un proceso de formación temprana con calidad en el que los maestros hagan uso de estrategias de evaluación formativa, realicen actividades de retroalimentación oportunas que garanticen procesos efectivos, y además integren a la familia, y que de esta forma la responsabilidad de la formación realmente se asuma desde ambas partes. Osorio y López (2014) plantean lo siguiente: 
El proceso académico de los estudiantes de preescolar depende en cómo los docentes le brindan al estudiante cierta comunicación para mejorar en sus desarrollos, logros y desempeños y en gran medida en cómo los padres de familia aprovecharon el informe para incrementar su compromiso y trabajo desde casa para avanzar en la formación de sus hijos. Por esta razón, se consideró importante comprender el rol de la retroalimentación en estudiantes en edad preescolar puesto que a través de esta se pueden lograr cambios en los procesos de enseñanza y aprendizaje. (p.14).

\section{El juego en la etapa del preescolar}

El juego es una actividad lúdica fundamental en el proceso de enseñanza y aprendizaje de los niños en la etapa del preescolar, el juego hace felices a los niños y los motiva hacia el aprendizaje, a través del juego se desarrollan o se fortalecen las diferentes capacidades del niño. Para Carrero y Martínez (2014) en la etapa del preescolar:

La actividad fundamental del niño es el juego. A través del juego de roles el niño comienza a comprender el mundo adulto con parte de su complejidad. El hecho que lo haga a través del juego le favorece la comprensión de la realidad y le disculpa los posibles errores ya que "se aprende jugando". La función del adulto es, por tanto, de estimulación, dirección y orientación de los participantes para que el juego de roles cumpla su cometido (p.6).

El juego es una práctica que desde los primeros años del individuo se lleva a cabo; con el juego los padres enseñan a caminar a sus hijos, los inducen cuando es necesario a generar buenos hábitos alimenticios, a reconocer su cuerpo; en fin, para el niño su vida es el juego. Según Imbacuán y Sotelo (2013) el juego es "una valiosa estrategia pedagógica y didáctica, para iniciar al niño y a la niña de edad preescolar en el proceso de construcción de 
conocimiento" (p.205). Con el juego el niño desarrolla su creatividad, se relaciona, de divierte, es feliz y al mismo tiempo aumenta la actividad congnitiva. A través del juego los niños aprenden a socializar, a contar, a asumir roles e incluso a entender el mundo de los adultos, el cual imitan de manera lúdica. Para Linaza (2013) "el juego es una necesidad es reconocer la forma específica con la que los niños abordan la realidad, sea física, social o intelectual" (p.104). Para Baena y Ruiz (2016)

El juego es un motor que constituye un elemento pedagógico de primer orden, ayudando a desarrollar la capacidad creativa y una mejor comprensión de los conceptos intrínsecos que subyacen en el lenguaje. También, facilita el desarrollo de los diferentes aspectos de la personalidad del niño/a, del carácter, habilidades sociales, dominios motores y capacidades físicas. Además, ofrece gran variedad de experiencias, lo cual facilita la adaptación y la autonomía (p.74).

A través del juego el niño en la etapa preescolar el niño se relaciona, especialmente con otros niños de su misma edad, comparte intereses afines, aprender a seguir directrices y normas, adquire pautas de comportamiento social. El juego en el preescolar no es simplemente una actividad didáctica, por el contrario, en esta etapa, según Vigotsky (1996, citado por Solovieva, Tejeda, García y Rojas, 2015, p.157), gracias al:

Juego se desarrolla la comunicación afectiva y verbal; la expresión verbal del niño se despliega, adquiere carácter voluntario, así como todas las demás actividades. El hecho de la existencia de roles que el niño tiene que expresar implica que el niño, al mismo tiempo, se somete a reglas culturales de comportamiento, es decir, su comportamiento pierde el carácter espontáneo e inmediato. (p.157). 


\section{El papel de la familia en el preescolar}

Para Loren y Reyes (2008) la familia es la primera escuela y los padres son los primeros educadores, por eso es necesario capacitarlos para que, con amor, eduquen integralmente a sus pequeños de manera actualizada. El adulto es quien organiza la vida del pequeño y satisface sus necesidades básicas: afecto, movimiento, alimentación, sueño, aseo, abrigo, lo estimula y le proporciona el contacto con las personas y con los múltiples objetos del mundo que le rodea, así como la realización de los diversos tipos de actividades que constituyen el centro de la educación en estas edades.

Teniendo en cuenta las teorías de los anteriores autores podemos analizar la importancia que representa el juego en los niños de preescolar, pero además de verlo como una simple actividad, es un proceso que conlleva también al relacionamiento del niño con su familia y con sus maestros, González y Mendoza (2015) opinan si los padres y educadores son capaces de observar a su hijo teniendo en cuenta que el juego es su vida, empezarán a ver el juego de una forma bien distinta a su creencia de que éste es pura diversión o una enfermedad del propio infante. Un juego educativo que hayamos comprado, puede no ser divertido y, si no hay diversión, difícilmente habrá aprendizaje. El infante sabe bien lo que le gusta y lo que no, y no le convenceremos de lo contrario. Es por tanto un preciado medio de educación, de ahí la importancia que reviste la preparación a la familia para el óptimo aprovechamiento de las potencialidades que este brinda en la formación desde edades tempranas de las nuevas generaciones.

Según Chacón (2005, citado por Jiménezy Araya, 2012) las investigaciones apoyan el papel fundamental que el juego posee en el desarrollo socioemocional, cognitivo y físico del niño, así como en la regulación de las emociones, el fortalecimiento de las relaciones sociales con los miembros de su familia y amigos, en la atención, en la resolución de problemas, en la creatividad y en el desarrollo de destrezas motoras gruesas y finas, así como en su salud en general. 
El tema de la etapa preescolar se ha tratado en múltiples estudios, pero más enfocando el papel de las instituciones educativas y de los maestros como responsables del proceso formativos de los niños, y en este proceso se ha identificado otro actor más importante que el mismo maestro, como es el de la familia. Hemos tratado del papel del maestro y de la forma de realizar la evaluación de los procesos en esta etapa y de la importancia de la retroalimentación pero cabe resaltar el rol primordial que los padres tienen en esta etapa del niño. Para Cardemil y Román (2016) la asistencia al jardín infantil incide a su vez en la educación y cultura familiar, de modo que es importante poder determinar de qué forma la asistencia de los infantes a esta nivel de educación formal fortalece las pautas de crianza que apoyan el desarrollo infantil en el hogar y transforma o mitiga aquellas que son menos positivas para ellos. Al respecto, una evaluación en este plano permitiría incidir de manera más precisa en factores de resiliencia que se juegan a nivel familiar, aprovechando que en este nivel educativo padres y madres se acercan más y mejor dispuestos a las instituciones que atienden a sus hijos e hijas pequeños.

El niño de preescolar debe recibir educación de todos los frentes que lo rodean es decir tal y como lo plantean Jaramillo, Osorio e Iriarte (2011) la atención educativa que deben recibir los niños preescolares debe entenderse como un sistema de interrelaciones intensas entre el niño y sus compañeros, entre el niño y su maestro, la familia, el ambiente y la comunidad. Concentrar la labor del preescolar en el niño no significa dejar la educación a merced de los deseos infantiles. Implica, por el contrario, una planificación rigurosa. Pero esta planificación no está ya basada en lo que los adultos creen que deben transmitir, sino en lo que sabemos que los niños pueden descubrir, en sus posibilidades reales de avanzar en la construcción del conocimiento.

En opinión de Llovera (1991, citado por Jaramillo, Osorio e Iriarte, 2011), la atención al niño preescolar se hace preventiva al definirla como el conjunto de acciones tendientes a proporcionar a cada uno la cantidad de estimulación necesaria para que desarrolle al máximo su potencial, con esto se logrará atender alguna ma- 
nifestación de tempranos desajustes. Esta actividad preventiva es muy importante en el período preescolar, y tanto la familia como la escuela son centros para organizarla como dos sistemas de influencias interactivas de las cuales los niños se nutren, en relación con ellas crecen y construyen estrategias para orientar su desarrollo.

Finalmente se puede concluir que el papel de la familia en los primeros años de la edad escolar de los niños es fundamental; en ningún momento la institución educativa tendrá que asumir o reemplazar la relación de la familia. Como lo plantea Woods Winnicott (1986, citado por Vergara y Vélez Vasco, 2016) la función del jardín de infantes no consiste en sustituir a una madre ausente, sino en complementar y ampliar el papel que solo la madre puede desempeñar en los primeros años de la vida del niño.

\section{Utilización de mediaciones}

tecnológicas en la etapa preescolar

Mucho se habla sobre si es apropiado o no utilizar herramientas tecnológicas para el proceso de enseñanza-aprendizaje con los niños de preescolar; según lo plantean Ortiz Vargas y Pérez Díaz (2015) se constata las bondades de los recursos tecnológicos dentro del aula y específicamente en transición y da un claro ejemplo de las transformaciones que se pueden llevar a cabo con ayuda de los recursos tecnológicos, en cuanto a competencias propias del preescolar como lo son las dimensiones del desarrollo humano. Para López Araujo (2013) en las horas de clase los niños cumplen con muchas actividades para desarrollar diferentes destrezas, cuando asisten al laboratorio de informáticas aunque no sepan leer y escribir, los niños desarrollan competencias en el uso de manejo de herramientas tecnológicas buscan gráficos, íconos con colores llamativos y representaciones con personajes.

Para algunos el uso de herramientas tecnológicas en niños de preescolar puede resultar preocupante, pero según Paniagua-Esquivel, Alfaro y Fornaguera (2016) se ha propuesto que es posible 
re direccionar su uso del computador en pro del aprendizaje, introduciéndola como un elemento cotidiano, como un instrumento de comunicación, de adquisición de información y para favorecer la interacción social.

Cabe destacar que las herramientas tecnológicas pueden resultar inofensivas con los niños de preescolar si se enfocan tal y como lo plantea Leyva, Pineda, Valencia y Oregón (2014) es evidente la importancia de crear situaciones de interacción de los niños de nivel preescolar con la tecnología, generar una cultura de tecnología bien estructurada, hacer énfasis en el dinamismo implícito en los medios electrónicos, pero sobre todo demostrar que la informática es una herramienta eficaz para el apoyo en el proceso enseñanza/ aprendizaje.

Para Sluis et al. (2004), Khandelwal y Mazalek (2007) y Tyng et al. (2011) (citados por Nácher Soler, 2014), demuestran que la tecnología puede ser utilizada por niños con edades entre tres y siete años para aprender habilidades de lectura, resolver problemas matemáticos, desarrollar el sentido del espacio, etc. Además, diversos estudios demuestran que esta tecnología también proporciona importantes resultados en la promoción de la colaboración entre compañeros y a la hora de fomentar su creatividad.

Argumenta Camargo y Orozco (2013). Por otra parte, reconociendo que es imposible detener la dinámica mundial relacionada con el acceso y uso de este tipo de dispositivos, en la actualidad se plantea la necesidad de utilizar los medios en forma positiva, para incrementar los hábitos alimenticios saludables y las conductas pro-sociales, y para disminuir el consumo de alcohol, el de tabaco y la iniciación temprana de relaciones sexuales. Además, se propone la necesidad de educar a los padres para controlar no solo el tiempo, sino el contenido y la calidad de los programas de televisión y de las redes de información a las que acceden sus hijos, así como de implementar normas en los hogares para su utilización, siguiendo las recomendaciones de las organizaciones internacionales. 


\section{Innovación educativa para preescolar}

La tecnología avanza de forma vertiginosa día a día; por ello utilizar todo tipo de mediaciones tecnológicas como estrategia de innovación para ayudar en los procesos educativos con los niños de preescolar es una tarea diaria de los maestros, instituciones educativas, y de los padres de familia, pero no buscando en ellas la solución a los problemas de aprendizaje, sino como factor motivacional, ya que nos encontramos ante niños con tendencias o preferencias diferentes relacionadas con las tecnologías que ocupan un papel importante hoy en el mundo. Para Escorsa (1997, citado por Reyes y Rivera, 2016), innovación es "el proceso en el cual a partir de una idea, invención o reconocimiento de una necesidad se desarrolla un producto, técnica o servicio útil hasta que sea comercialmente aceptado", y desde luego social y culturalmente admitido.

Según Reyes y Rivera (2016) para innovar o crear es indispensable el conocimiento del contexto donde se desarrolla la idea, con miras a construir estrategias novedosas, donde se integren los recursos tecnológicos, escapando al ambiente educativo tradicional y convencional buscando un impacto transformador en la comunidad educativa; se considera que es desde el contexto escolar, donde se eliminan las brechas sociales y la desigualdad en la utilización, acceso e implementación de los recursos tecnológicos en los procesos de aprendizaje y en la vida diaria, fomentando desde allí su uso y adecuada utilización, buscando con esto acercar e incorporar las Tecnologías de la Información y de la Comunicación (TIC) a la realidad de los estudiantes desde los primeros grados de escolaridad.

Plantea Solórzano Castro (2015) existe un sin número de software diseñados para el nivel inicial, los mismos que comprenden actividades que utilizadas de manera adecuada y en espacios propicios no solo desarrollarían y fortalecerían habilidades y destrezas, sino que, también darían la oportunidad de fomentar valores y hábitos de convivencia escolar, ya que los y las niñas aprenderían de manera divertida a socializar, compartir y a respetar a través de 
juegos, canciones y demás actividades que generan el aprendizaje significativo.

Para Dixon, NiMhurchu, Meagher y Widdowson (2010, citados por Jiménez y Araya, 2012) existe evidencia que indica que tanto los padres como los hijos consideran que los videojuegos activos promueven la actividad física y pueden contribuir a reducir la obesidad infantil. Para Jiménez y Araya (2012) jugar un videojuego activo es más beneficioso que ser sedentario. Sin embargo, los videojuegos activos son más beneficiosos que los videojuegos pasivos, pero vale la pena decir el juego tradicional y el proporcionado por los videojuegos seguirá siendo importante en todas las etapas del desarrollo de niños y adolescentes.

Según Salinas (2008, citado por Pinto Torres, 2016), de hecho, los procesos de innovación educativa coinciden con un proceso de cambio, construcción y participación social que implica repensar contenidos establecidos, estrategias, metodologías y proponer ambientes de aprendizaje con mayor efectividad. Lo que implica para el caso de los niños de preescolar el uso de mediaciones tecnológicas como estrategia de innovación no implica simplemente diseñar plataformas, juegos o video juegos, entre otros, se hace necesario hacer investigaciones que permitan determinar la incidencia que pueden tener estos procesos en los procesos físicos o cognitivos de los niños.

Los maestros relacionados con los niños del nuevo milenio deben ser mediadores, facilitadores, guías y sobre todo orientadores, las mediaciones tecnológicas son una realidad en el proceso formativo de los niños por lo tanto tal y como lo plantea Fernández (2016) Los expertos manifiestan que el maestro de este nuevo milenio deberá abordar nuevas tareas y desafíos, haciéndose necesaria una actitud abierta a los múltiples acontecimientos e informaciones que se generan a su alrededor, desarrollando habilidades para flexibilizar la incorporación a su práctica profesional de los distintos recursos que les aporten los sucesivos cambios tecnológicos. De manera tal, que el profesor se convierte en la pieza clave para la integración de las tecnologías en el ámbito educativo, 
necesitando sobre todo formación y actitudes positivas hacia estas nuevas herramientas. Debe conocerlas, aprender a manejarlas, conocer sus potencialidades como instrumento pedagógico y saber cómo seleccionarlas, cómo introducirlas en el aula y cómo evaluar su uso.

A juicio de Gutiérrez (1996, citado por Fernández, 2016) la utilización de estas nuevas tecnologías en la enseñanza debe servir al profesor para superar el modelo comunicativo unidireccional y no reforzarlo, con lo que se debe preocupar en que el alumno aprenda a utilizar la tecnología como medio de expresión y deje de considerarse como un mero receptor de la información. En ese contexto, la presencia de las tecnologías en el ámbito educativo, puede ser aprovechada para el logro de dos grandes finalidades: la de educar con los medios y la de formar en los medios; en ambos casos la misión es la de desarrollar una criticidad y reflexividad frente a esta nueva realidad hacia el alumnado. Sin embargo, la simple presencia de las TIC en el aula no asegura el éxito ni la calidad de la enseñanza, descansando, en buena medida su utilidad, en el papel del propio profesor y la relación que establezca con el alumnado.

Todo esto implica una responsabilidad compartida: las tecnologías por sí solas no son ni buenas ni malas como apoyo en los procesos formativos de los niños, pero si van de la mano del uso correcto y efectivo por los maestros y los padres de familia, realmente se convierten en una estrategia educativa innovadora, que además de ser motivadora puede contener un amplio portafolio de oportunidades, donde cada día el niño, maestros y padres de familia encuentren en plataformas, videojuegos, páginas web, entre muchos otros espacios virtuales, una variada gama de oportunidades de aprender de forma divertida.

\section{Metodología}

La orientación del diseño metodológico de la investigación corresponde a un estudio descriptivo-cuantitativo, no experimen- 
tal, con toma de datos longitudinal. Se seleccionó una institución educativa pública del municipio de Bello, la cual tiene en el nivel de preescolar 3 grupos de transición con el siguiente número de estudiantes: grupo 1 (30 estudiantes), grupo 2 (25 estudiantes), grupo 3 (25 estudiantes). Para la muestra se seleccionaron padres o acudientes, con un nivel de confianza del $95 \%$, margen de error de $5 \%$. Se establecieron valores probabilísticos de $p: 0,7$ y $q: 0,1$, valores de $p$ y $q$ que para el cálculo de la muestra se encontraron por prueba piloto; con estos parámetros se realizó el cálculo y este arrojó como resultado 67 . En cuanto a los expertos infantiles se contó con una muestra seleccionada de forma no aleatoria, definida de manera selectiva por criterio (conveniencia) con un total de 50 especialistas. Los maestros participantes fueron los asignados por la institución como directores de los 3 grupos.

Se utilizaron dos tipos de instrumentos para la recolección de la información: en primer lugar, se diseñó una encuesta estructurada para recolectar información de los maestros de preescolar, y posteriormente se aplicó la técnica de la entrevista a especialista infantiles, como pedagogos y psicólogos, en el año 2016; y la segunda entrevista se aplicó en el año 2017 a acudientes de niños de preescolar, entre padres y abuelos. Los datos fueron recolectados por los investigadores y el estudio se realizó en los años 2016 y 2017.

Figura 1. Apreciación de profesores sobre los videojuegos como instrumento de apoyo al proceso de enseñanza-aprendizaje.

Videos juegos instrumento de apoyo al proceso de enseñanza-aprendizaje

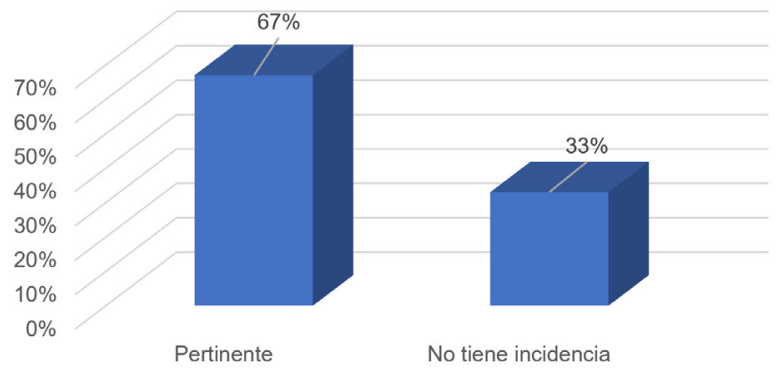

Fuente: elaboración propia. 
La figura 1 permite evidenciar la apreciación de los profesores de preescolar encuestados con respecto a utilizar videojuegos como instrumento de apoyo al proceso de enseñanza-aprendizaje, respecto a lo cual la mayoría, que equivale al $67 \%$, consideran que resulta pertinente, y el $33 \%$, no tiene incidencia; lo cual significa que validan el uso de estos instrumentos como una experiencia que no tiene incidencias negativas en los procesos cognitivos de los niños.

Figura 2. Apreciación sobre complementar el método tradicional con ambientes virtuales.

Complementar el método tradicional con ambientes virtuales

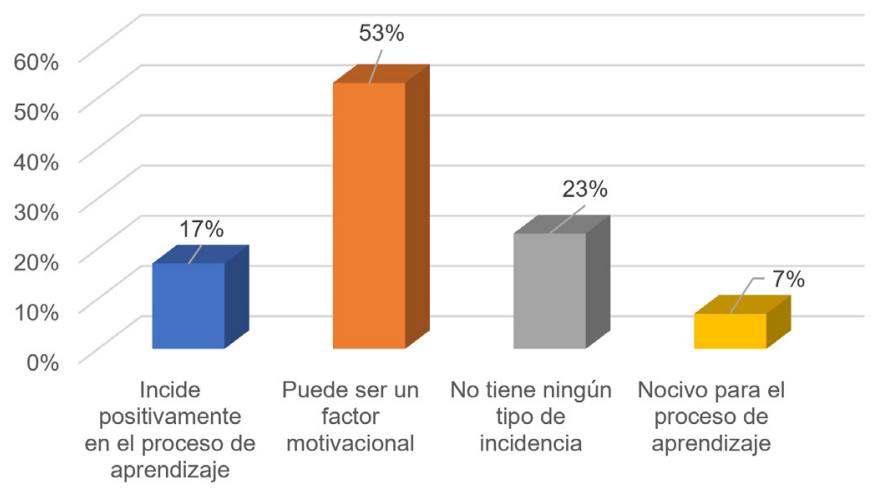

Fuente: elaboración propia.

Se evidencia en la figura 2 que el $53 \%$ de los especialistas entrevistados consideran que complementar el método tradicional con ambientes virtuales puede ser un factor motivacional para el aprendizaje de los niños de preescolar; además, el $17 \%$ también consideran que es un proceso que incide positivamente, debido a que son herramientas a las cuales están acostumbrados. 
Figura 3. Impacto de la tecnología en los nativos digitales.

Impacto de la tecnología e los nativos digitales

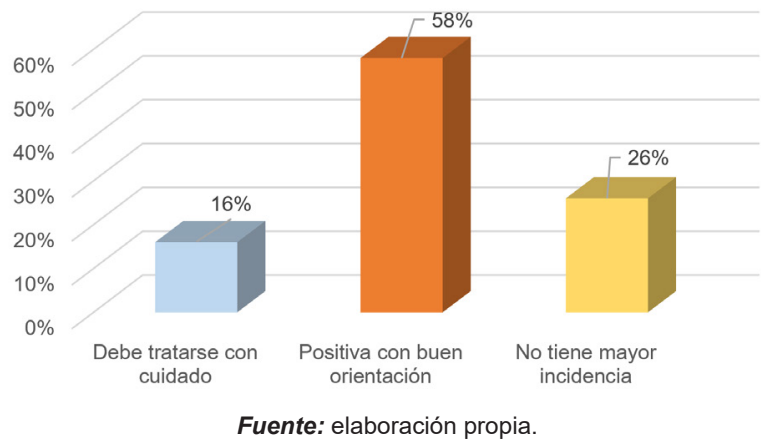

La figura 3 muestra que la mayoría de los especialistas infantiles, que equivale al $58 \%$, consideran que el impacto sobre el uso de herramientas tecnológicas con los niños es positivo, siempre que se tenga en cuenta que debe usarse bajo una adecuada orientación y con precaución; de lo contrario, puede resultar nocivo para el aprendizaje de los niños.

Figura 4. Procesos en los que utilizan mediaciones tecnológicas.

Procesos en los que utilizan mediaciones tecnológicas

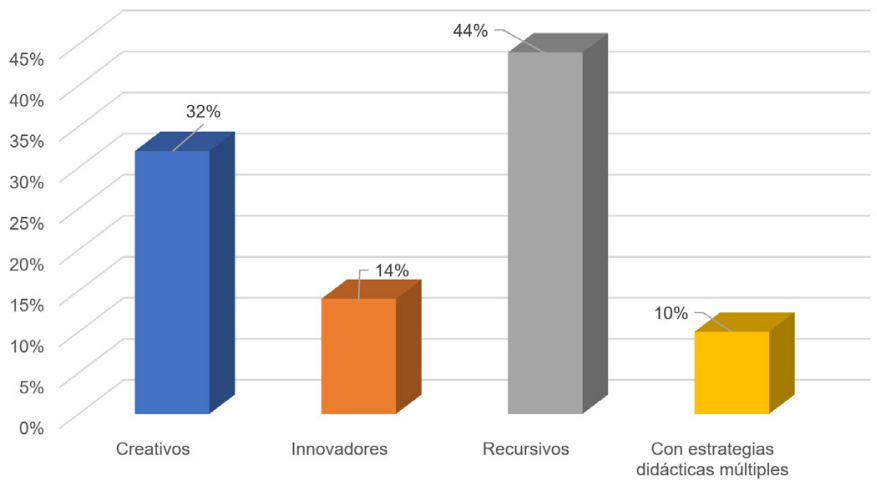

Fuente: elaboración propia.

La figura 4 muestra la opinión de los especialistas con respecto a los procesos en que los maestros hacen uso de mediaciones tecnológicas en el aula con los niños de preescolar, considerándolos en su mayoría como procesos recursivos y creativos. 
Figura 5. Utilizar las TIC como apoyo formativo del niño.

Utilizar las TIC como apoyo formativo del niño

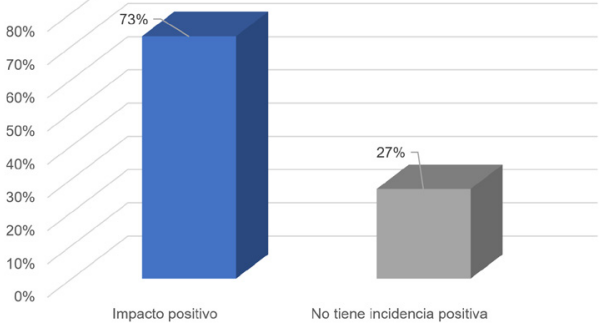

Fuente: elaboración propia.

La figura 5 muestra la opinión de los acudientes con respecto a utilizar las TIC como herramientas de apoyo en el proceso formativo de los niños de preescolar; la mayoría, que equivale al $73 \%$, consideran que genera un impacto positivo, lo que significa que para un alto porcentaje de los acudientes el uso de las TIC tiene aceptación en el proceso de enseñanza-aprendizaje con los niños.

Figura 6. No tiene incidencia positiva.

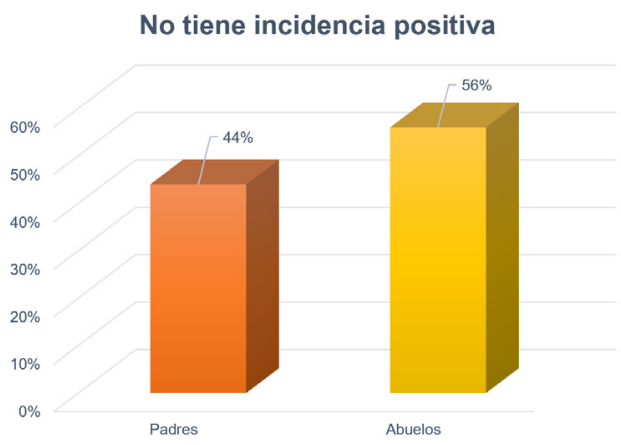

Fuente: elaboración propia.

La figura 6 tiene relación directa con la figura 5 porque muestra cómo se componen los acudientes para los cuales el uso de TIC no tiene incidencia positiva; es importante destacar que dentro de ese grupo de acudientes el mayor porcentaje, que equivale al 56 $\%$, son los abuelos, personas mayores de 60 años para las cuales es normal en su vida cotidiana no hacer uso de este tipo de herramientas tecnológicas. 
Figura 7. Uso de dispositivos tecnológicos para jugar con el niño en su tiempo libre.

Uso de dispositivos tecnológicos para jugar con el niño en su tiempo libre

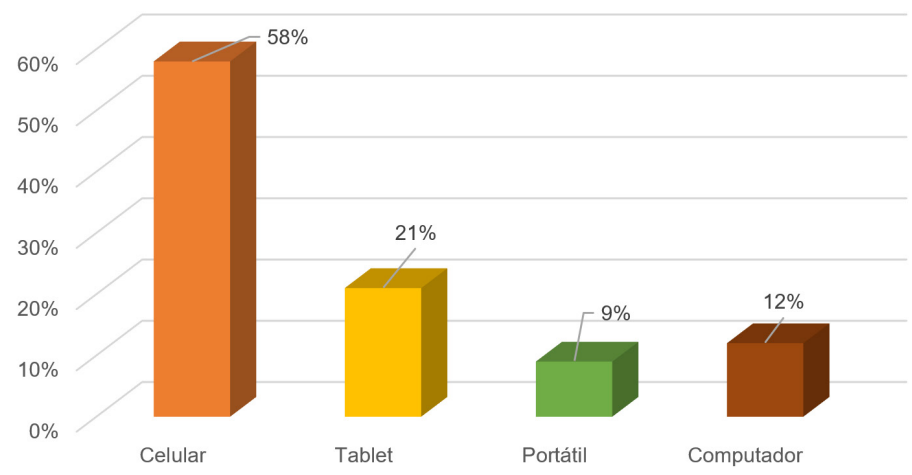

Fuente: elaboración propia.

La figura 7 muestra que el celular es el dispositivo más utilizado por los acudientes para jugar con los niños en espacios fuera del aula de clase, es decir, en su tiempo libre, y en segundo lugar tiene relevancia de uso la tablet. El computador personal y el portátil tienen menor frecuencia de uso, sin embargo, siguen siendo utilizados.

Figura 8. Utiliza el niño con frecuencia alguna aplicación virtual.

Utiliza el niño con frecuencia alguna aplicación virtual

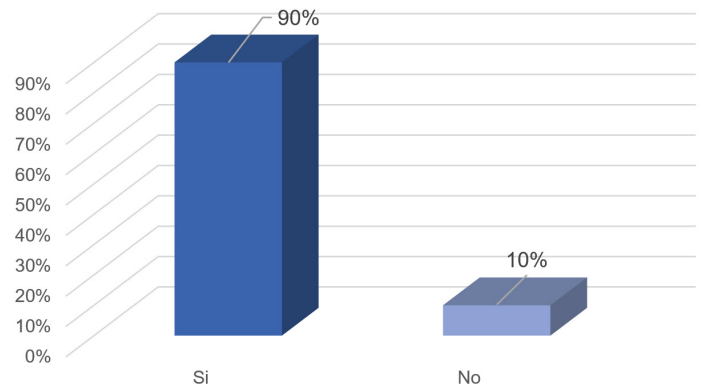

Fuente: elaboración propia. 
La figura 8 muestra que el $90 \%$ de la población entrevistada (acudientes) afirman que el niño utiliza con frecuencia alguna aplicación virtual; como esto lo indica, los niños encuentran entretenido el uso de ambientes virtuales para su diversión y, por ende, sus procesos de aprendizaje.

Figura 9. Aplicaciones digitales utilizadas por el niño con frecuencia.

$$
\begin{aligned}
& \text { Aplicaciones digitales utilizadas por el niño con } \\
& \text { frecuencia }
\end{aligned}
$$

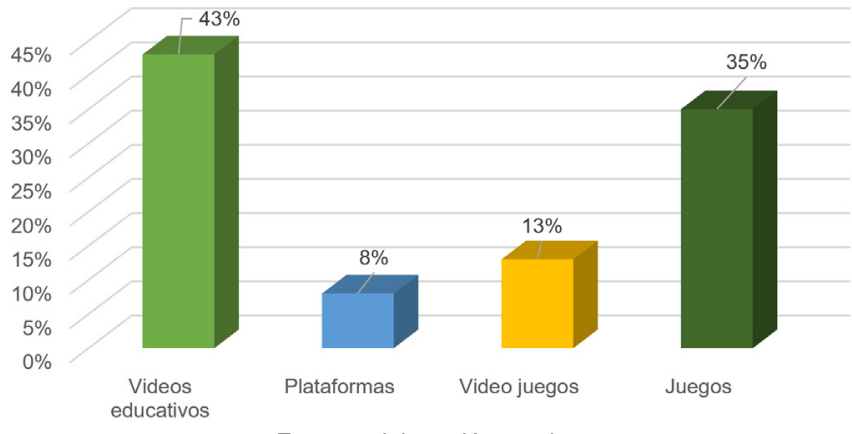

Fuente: elaboración propia.

La figura 9 tiene relación directa con la figura 8, sobre la población cuyos niños utilizan con frecuencia alguna aplicación virtual; a ellos se les preguntó cuáles son las más utilizadas o frecuentadas por los niños; el 43 \% responde que los videos educativos, y en segundo lugar, equivalente al $35 \%$, que utiliza juegos.

Figura 10. Utilizaría plataformas de juegos para apoyar el aprendizaje de su niño. aprendizaje de su niño

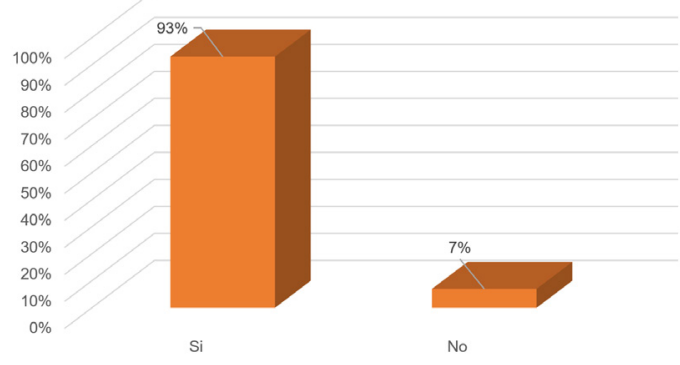

Fuente: elaboración propia. 
Evidentemente, en la figura 10 se muestra que hay una fuerte aceptación, por un alto porcentaje de la población entrevistada, con respecto a utilizar una plataforma que mediante el uso de juegos permita apoyar el proceso de aprendizaje de los niños; proceso que, además de ser innovación educativa, es un factor motivacional para padres, maestros y niños.

\section{Discusión}

Tal y como lo plantea Escorsa (1997, citado por Mora y Morales, 2016), innovación es "el proceso en el cual a partir de una idea, invención o reconocimiento de una necesidad se desarrolla un producto, técnica o servicio útil hasta que sea comercialmente aceptado, y desde luego social y culturalmente admitido" (p.118); los niños de hoy, como nativos digitales, están rodeados de tecnología y más aún acostumbrados a programas de televisión, a videos y juegos digitales más que a otro tipo de prácticas cotidianas que las generaciones anteriores; es por eso que bajo esta realidad tomar la tecnología como una innovación direccionada en el factor educativo realmente es una estrategia viable.

Para muchos autores, el uso de mediaciones tecnológicas puede ser una práctica no muy positiva, pero otros, como Paniagua-Esquivel, Alfaro y Fornaguera (2016) "se ha propuesto que es posible re direccionar su uso del computador en pro del aprendizaje, introduciéndola como un elemento cotidiano, como un instrumento de comunicación, de adquisición de información y para favorecer la interacción social" (p.425). Para el caso de los niños en etapa preescolar, tal y como lo afirman Sluis et al. (2004), Khandelwal y Mazalek (2007) y Tyng et al. (2011) (citados por Nácher [2014]), se ha demostrado que la tecnología puede ser utilizada por niños con edades entre tres y siete años para aprender habilidades de lectura, resolver problemas matemáticos, desarrollar el sentido del espacio, etc. Además, diversos estudios demuestran que esta tecnología también proporciona importantes resultados en la promoción de la colaboración entre compañeros y a la hora de fomentar su creatividad. 
De acuerdo a la investigación realizada, los resultados obtenidos muestran que el uso de tecnología con aplicaciones digitales es una realidad ante la cual no podemos negarnos; los niños de hoy nacieron rodeados de este tipo de estos ambientes virtuales, por lo cual para protegerlos ante posibles efectos negativos que generen el uso de juegos, videojuegos o cualquier ambiente virtual la clave no es prohibirlos y a toda costa evitar su uso; por el contrario, es aquí donde resulta importante la presencia y el debido acompañamiento de la familia, los maestros y la sociedad en general, quienes intervienen en el proceso formativo de los niños. Educarlos en el uso correcto de ellos sirve para generar cultura desde temprana edad, a través de asegurar que se aprenda el uso correcto y efectivo de la tecnología como apoyo al aprendizaje y sobre todo como factor motivacional y lúdico.

No solo es deber de los maestros, investigadores, psicólogos, y de todo tipo de profesionales velar por la protección de los niños, sino que es obligación de toda la familia cuidar y supevisar la educación sana de los niños. Tal como lo plantean Jaramillo, Osorio e Iriarte (2011) "la atención educativa que deben recibir los niños preescolares debe entenderse como un sistema de interrelaciones intensas entre el niño y sus compañeros, entre el niño y su maestro, la familia, el ambiente y la comunidad" (p.155). La función del preescolar implica diseñar, planear acciones y actividades acordes a su edad y relacionadas con su proceso de aprendizaje y es así como podemos integrar de forma responsable el uso de mediaciones tecnológicas digitales que permitan complementar desde las diferentes dimensiones el sano desarrollo de los niños, utilizando el juego (videojuegos) como eje transversal en los procesos de enseñanza y aprendizaje.

\section{Conclusiones}

En el nivel preescolar, es decir entre los 3 y 5 años de vida, el niño está en una edad de descubrimiento, inventos, curiosidades y de desarrollo de tipos de conducta sociocultural, lo que implica la 
oportunidad para maestros y padres de familia de generar procesos de correcta cultura con respecto al eficiente y efectivo uso de la tecnología para impactar positivamente en los procesos de aprendizaje de los niños; es una realidad hoy que los niños tienen a su alrededor herramientas tecnológicas, lo importante es enseñarles su uso adecuado y aprovechar las fortalezas que estas tienen para su aprendizaje significativo.

Concentrar la labor del preescolar en el niño no significa dejar la educación a merced de los deseos infantiles, dejarlo utilizar herramientas tecnológicas para jugar sin la debida supervisión puede resultar nocivo para el proceso de aprendizaje del niño; darle un dispositivo tecnológico solo para que él se entretenga no es una actitud responsable, lo importante de la presencia de la tecnología en el proceso de exploración de los niños es que les permite conocer, por medio de maestros, padres de familia y demás adultos que los rodean, las diferentes alternativas que ofrece la tecnología para aprender de forma divertida y dinámica.

El juego, además de ser una necesidad en el ser humano, se convierte en un factor motivacional, más aún en los niños en la etapa preescolar, cuando a través de él se pueden desarrollar las diferentes dimensiones del ser humano; pero es importante relacionar este tipo de vivencias con los ambientes naturales para los niños de hoy, como son los ambientes tecnológicos. Cada ves resulta más evidente la importancia de crear situaciones de interacción de los niños de nivel preescolar con la tecnología, pero sobre todo demostrar que la informática es una herramienta eficaz para el apoyo en el proceso enseñanza-aprendizaje, y sobre todo con el acompañamiento y supervisión de maestros y familiares. 


\section{Referencias bibliográficas}

Baena, A y Ruiz, P. J. (2016). El juego motor como actividad física organizada en la enseñanza y la recreación. EmásF: Revista Digital de Educación Física, 38, 73-86.

Camargo, D. M. y Orozco, L. C. (2013). Factores asociados a la disponibilidad y uso de medios electrónicos en niños desde preescolar hasta 4. grado. Biomédica, 33(2), 175-185.

Cardemil, C. y Román, M. (2014). La importancia de analizar la calidad de la educación en los niveles Inicial y Preescolar. Revista Iberoamericana de Evaluación Educativa, 7(1), 9-11.

Carrero, M. Z. y Martínez, M. A. (2014). Importancia del preescolar en el desarrollo psicológico, creativo y social de los niños de III nivel, Colegio Rubén Darío del barrio Guadalupe-León, I semestre 2014 (Doctoral dissertation). Universidad Nacional Autónoma de Nicaragua, León, Nicaragua.

Fernández, C. (2016). Elementos que contribuyen a la construcción de un modelo curricular para la integración de TIC en el segundo ciclo de educación parvularia. REXE: Revista de Estudios y Experiencias en Educación, 10(20), 109-125.

González, Y. B. y Mendoza, Y. M. (2015). Acciones educativas para la preparación de la familia en el desarrollo de juegos en el hogar. Lecturas: Educación Física y Deportes, 202, 2.

Imbacuán, E. C. L., \& Sotelo, A. D. (2013). El juego como generador de aprendizaje en preescolar. Revista criterios, 20(1), 203-218.

Jaramillo, L. D. L., Osorio, M. e Iriarte, F. (2011). Reflexiones en torno al acompañamiento en los procesos de mejora de la práctica educativa en la educación preescolar. Zona Próxima, 15, 150-163. 
Laureano, G. (2015). Prácticas de crianza de los padres de preescolares que asisten al control de crecimiento y desarrollo del centro de salud Nueva Esperanza, Villa María del Triunfo, Lima - 2014. (nivel universitario), Universidad Nacional Mayor de San Marcos, Lima; Perú.

Linaza, J. L. (2013). El juego es un derecho y una necesidad de la infancia. Bordón: Revista de Pedagogía. 65(1). 101-118.

López, M. A. (2013). Creación de recursos interactivos en lectura para niños de educación preescolar del colegio Thomas Jefferson, utilizando técnicas de ilustración y animación digital. (nivel tecnológico), Universidad de las Américas, Santiago, Chile.

Loren, N. y Reyes, E. (2008). La actividad conjunta: una forma de preparar a la familia en el Programa Educa a tu Hijo. Revista EduSol, 8(25). 80-89.

Moncada, J y Chacón, Y. (2012). El efecto de los videojuegos en variables sociales, psicológicas y fisiológicas en niños y adolescentes. Retos: Nuevas Tendencias en Educación Física, Deporte y Recreación, 21, 43-49.

Mora, J. Z. y Morales, S. P. (2016). Fortalecimiento en los procesos lecto-escritos en primera infancia a través de blended-learning. REICE: Revista Electrónica Iberoamericana sobre Calidad, Eficacia y Cambio en Educación, 14(1), 117-135.

Nácher, V. E. (2014). Estudio de las capacidades motoras de niños en edad preescolar para la realización de gestos en superficies multitáctiles. (nivel máster), Universitat Politècnica de València, Valencia, España.

Ortiz Vargas, S. N. y Pérez Díaz, L. K. (2015). Análisis de la apropiación de las TIC en los docentes en nivel de transición de las instituciones educativas privadas del municipio de Barbosa, Santander (trabajo de grado). Universidad Pedagógica y Tecnológica de Colombia, Tunja.

Osorio, K. y López, A. (2014). La retroalimentación formativa en el proceso de enseñanza-aprendizaje de estudiantes en edad preescolar. Revista Iberoamericana de Evaluación Educativa, 7(1). 13-30. 
Paniagua-Esquivel, C., Alfaro, R. y Fornaguera, J. (2016). Aporte docente en el diseño de Ambientes Virtuales Colaborativos para educación preescolar. Ciencia, Docencia y Tecnología, 27(52), 423-440.

Pinto Torres, R. M. (2016). Innovación educativa mediada por TIC, una oportunidad de enriquecer la labor pedagógica del docente. (nivel maestría), Universidad de la Sabana, Cundinamarca, Colombia.

Solórzano Castro, B. A. (2015). Las NTIC's y su impacto en la educación de los niños y niñas en edad preescolar del Centro Integral del Buen Vivir "Sonrisitas" del barrio Mariscal Sucre, cantón La Libertad, provincia Santa Elena, período lectivo 2013-2014. (nivel universitario), Universidad Estatal Península de Santa Elena, La Libertad, Ecuador.

Solovieva, Y., Tejeda, L., García, E. L. y Rojas, L. Q. (2015). Propuesta para el uso del juego de roles en la institución preescolar. Educação e Filosofia, 29(57), 153-174.

Torres, J., Ortiz, Cuevas, R. E. y Gómez, M. (2014). Educando a los nativos digitales de preescolar con apoyo de herramientas didácticas de software libre. Vínculos, 10(2), 421-434.

Vergara, C. E. y Vélez, I. C. (2016). La adaptación escolar: una experiencia investigativa en preescolar. En-Clave Social, 5(1), 8-16. 